This item was submitted to Loughborough's Research Repository by the author.

Items in Figshare are protected by copyright, with all rights reserved, unless otherwise indicated.

\title{
Logical relativism: logic, grammar, and arithmetic in cultural comparison
}

PLEASE CITE THE PUBLISHED VERSION

http://dx.doi.org/10.1353/con.0.0016

\section{PUBLISHER}

(c) The Johns Hopkins University Press and the Society for Literature and Science

\section{VERSION}

AM (Accepted Manuscript)

\section{LICENCE}

CC BY-NC-ND 4.0

\section{REPOSITORY RECORD}

Greiffenhagen, Christian, and Wes Sharrock. 2019. "Logical Relativism: Logic, Grammar, and Arithmetic in Cultural Comparison”. figshare. https://hdl.handle.net/2134/14206. 
This item was submitted to Loughborough's Institutional Repository (https://dspace.lboro.ac.uk/) by the author and is made available under the following Creative Commons Licence conditions.

\section{creative
commons}

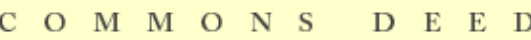

Attribution-NonCommercial-NoDerivs 2.5

You are free:

- to copy, distribute, display, and perform the work

Under the following conditions:

Attribution. You must attribute the work in the manner specified b the author or licensor.

Noncommercial. You may not use this work for commercial purposes.

No Derivative Works. You may not alter, transform, or build upon this work.

- For any reuse or distribution, you must make clear to others the license terms of this work.

- Any of these conditions can be waived if you get permission from the copyright holder.

Your fair use and other rights are in no way affected by the above.

This is a human-readable summary of the Leqal Code (the full license).

\section{Disclaimer 만}

For the full text of this licence, please go to: http://creativecommons.org/licenses/by-nc-nd/2.5/ 


\title{
Logical Relativism:
}

\section{Logic, Grammar, and Arithmetic in Cultural Comparison}

Authors: Christian Greiffenhagen and Wes Sharrock

\author{
Address: \\ Christian Greiffenhagen \\ Department of Sociology \\ Arthur Lewis Building \\ University of Manchester \\ Manchester M13 9PL \\ UK
}

Email: Christian.Greiffenhagen[replace_with_@]manchester.ac.uk

Version: June 14, 2008

Greiffenhagen, C. and W. Sharrock (2006). Logical relativism: logic, grammar, and arithmetic in cultural comparison. Configurations 14 (3), 275-301. 


\title{
Logical Relativism:
}

\section{Logic, Grammar, and Arithmetic in Cultural Comparison}

\begin{abstract}
Logical relativism is the claim that different cultures may think according to different logical laws. For example, it is often argued that whereas 'we' (Westerners) operate according to the law of the excluded middle, 'they' (e.g., the Chinese or the Azande) may not. In this article, we question whether logical relativism is an empirical thesis, i.e., a thesis that is substantiated through anthropological examples.
\end{abstract}

We distinguish two forms of logical relativism, both of which try to account for alleged contradictions in the beliefs of other cultures. The 'alternative logic' approach suggests that contradictions only appear if we judge beliefs according to classical logic, but do not exist if we judge them according to an alternative (non-standard) logic. The 'symmetric treatment' suggests that whether there is a contradiction or not is itself a culture-specific matter, such that what may be a contradiction 'for them' may not be a contradiction 'for us' and vice versa. We question whether either of these arguments really involves relativism and show that problems arise in the treatment of the examples, firstly, in terms of questionable preconceptions made about the status of logic as a standard of comparison and, secondly, in the ways in which relevant beliefs are formulated.

Greiffenhagen, C. and W. Sharrock (2006). Logical relativism: logic, grammar, and arithmetic in cultural comparison. Configurations 14 (3), 275-301. 


\section{Introduction}

In this article we question the plausibility of logical relativism, which we take to be the claim that people in other cultures may follow, believe in, or subscribe to a different form of logic from 'our' Western logic ${ }^{1}$. In other words, logical relativism argues that classical logic (including the law of non-contradiction [LNC] and the law of the excluded middle [LEM]) rather than being universal may only be locally true, i.e., applicable only within a culture's limits. The question whether every culture accepts the LEM is part of longrunning arguments about the rationality of different cultures. Since it is typically assumed that 'we' (Westerners) accept the LEM, the existence of a culture that did not subscribe to the LEM would, it has been argued, demonstrate either (a) that that culture falls short of the universal standard of rationality (since they do not reason according to classical logic) or (b) that the culture is nonetheless rational, but that the laws of classical logic are not a universal standard of rationality.

Logical relativism is often seen as being supported or indeed inspired by comparative studies that demonstrate that other cultures do not conform to classical logic. For example, the Azande ${ }^{2}$ are sometimes seen as eschewing the LEM, as a result of the following beliefs: The Azande believe that witchcraft is hereditary. Since clan members

\footnotetext{
${ }^{1}$ This paper is the third and final part of a project in which we investigate the empirical evidence for arguments about the cultural relativity of logic, grammar, and arithmetic; see "Mathematical Relativism: Logic, Grammar, and Arithmetic in Cultural Comparison”, Journal for the Theory of Social Behaviour 36:2 (2006): 97-117, and "Linguistic Relativism: Logic, Grammar, and Arithmetic in Cultural Comparison”, Language \& Communication 27:1 (2007): 81-107.

${ }^{2}$ E. E. Evans-Pritchard, Witchcraft, Oracles and Magic among the Azande (Oxford: Clarendon, 1937).
}

Greiffenhagen, C. and W. Sharrock (2006). Logical relativism: logic, grammar, and arithmetic in cultural comparison. Configurations 14 (3), 275-301. 
are related, and this is a small community, this would seem to entail that as soon as one clan member is identified as a witch, all clan members are thereby witches. However, the Azande also believe that a clan may have members that are, and members that are not, witches. Taken together, these beliefs seem to implicate the Azande in a contradiction. The question of logical relativism thus seems to be an empirical one, to be demonstrated (or possibly refuted) by anthropological studies of how other cultures 'think':

\begin{abstract}
Relativistic views about logic have surfaced in the works of social anthropologists, cognitive scientists, and philosophers. Reports by anthropologists about the thinking habits of remote peoples have led to the suggestion that rules of logic may have only a local rather than universal authority. $^{3}$
\end{abstract}

Can one without further ado simply assume that all the people the anthropologists have canvassed subscribe to the LEM? If one could, that would greatly strengthen the case for the universality of logical principles. The problem is that the Azande, for example, have been taken to be a people who rejected it. [...] it becomes an empirical question which available set of logical principles best accords with a practice of a particular people. A particular set of logical rules then becomes a model for that specific style of reasoning. ${ }^{4}$

In this paper we question the sense in which it is "an empirical question which available set of logical principles best accords with a practice of a particular people”. We neither argue that logical principles are universal, nor that they are relative. Rather, we

\footnotetext{
${ }^{3}$ Maria Baghramian, Relativism (London: Routledge, 2004); p.155; our emphasis.

${ }^{4}$ Diederick Raven, “The Enculturation of Logical Practice”, Configurations 4:3 (1996): 381-425; pp. 412 413; our emphasis.
}

Greiffenhagen, C. and W. Sharrock (2006). Logical relativism: logic, grammar, and arithmetic in cultural comparison. Configurations 14 (3), 275-301. 
investigate the role that logical principles are given in accounts of cultural practices (both 'at home' and 'away') and we question whether there is much sense in trying to characterise the way a whole culture 'thinks' in terms of formal logical systems.

Logical relativism is often justified as a reaction against an earlier Western cultural imperialism according to which other cultures were seen as less rational than Western cultures $^{5}$. Against this supposed imperialism of Western rationality, two slightly different forms of logical relativism emerged, one arguing for an 'alternative logic' in other cultures, the other arguing for a 'symmetric treatment' of the examples.

With respect to the Azande, some people have argued that although there may be a contradiction in Zande beliefs, this contradiction only appears if we investigate their beliefs according to classical logic. If we evaluate their beliefs according to an alternative logic, then the contradiction disappears: "Primitive magico-religious thought incorporates an alternative logic to our 'standard' one within the terms of which the apparent inconsistencies are not inconsistencies at all." ${ }^{6}$ According to this argument, the Azande are as logical as we are, but they reason according to different logical forms than we do. In other words, every culture thinks according to some formal logic, but 'our' classical

\footnotetext{
${ }^{5}$ It is difficult to find concrete examples of this view. Lucien Lévy-Bruhl's Primitive Mentality (London: George Allen \& Unwin, 1923; originally published in French in 1922 as La mentalité primitive) is sometimes seen as an example, but he wrote later in The Notebooks on Primitive Mentality (Oxford: Blackwell, 1975; originally published in French in 1949 as Carnets): "I asked myself whether societies of different structure had not also, ipso facto, specifically different logics (for example, the idea of a peculiarly Chinese logic distinct from western logics). - I had quickly renounced this hypothesis which at one and the same time simplistic and rather crude.” (p.48).

${ }^{6}$ David E. Cooper, “Alternative Logic in ‘Primitive Thought’”. Man 10:2 (1975): 238-256; p. 241.
}

Greiffenhagen, C. and W. Sharrock (2006). Logical relativism: logic, grammar, and arithmetic in cultural comparison. Configurations 14 (3), 275-301. 
logic (in particular the LEM) ceases to be an universal standard, since it is only one of many possible standards of rationality.

The symmetric relativist affirms that there is a contradiction in Zande thought, but points out that comparable contradictions also appear in Western thought. An example that has been given is that in our society murderers are those who deliberately kill people, which is seemingly in contradiction with the fact that bomber pilots are usually not seen as murderers (even though they deliberately kill people). Perhaps ‘we’ don’t treat bomber pilots as murderers, but it would not be irrational for a Zande anthropologist to condemn us for an inconsistency. In other words, it might be that what was a contradiction 'for us' may not be a contradiction 'for them' - and vice versa. While Evans-Pritchard sees a contradition in Zande thought which the Azande themselves do not see $^{7}$, an alien anthropologist visiting our culture could argue that he or she sees a contradiction in our treatment of fighter pilots that we ourselves do not see. The symmetric relativist concludes that "the Azande think very much as we do"8 and that the question whether there is a contradiction in a culture's beliefs is determined by which beliefs the particular group endorses. Consequently, logic is culturally relative, since cultures differ over whether something is a contradiction.

Both versions of logical relativism argue that whether something is a contradiction or not cannot be judged according to universal criteria, but only according to those logical standards indigenous to each culture. Our aim in this paper is to examine empirical

\footnotetext{
${ }^{7}$ Richard C. Jennings, “Zande Logic and Western Logic”, British Journal for the Philosophy of Science 40:2 (1989): 275-285; p. 281.

${ }^{8}$ David Bloor, Knowledge and Social Imagery (London: Routledge, 1976); p. 129.
}

Greiffenhagen, C. and W. Sharrock (2006). Logical relativism: logic, grammar, and arithmetic in cultural comparison. Configurations 14 (3), 275-301. 
examples of the kind that both types of logical relativists take as their starting point. Both sides operate as if it is relatively easy to establish what it is that is believed in the relevant cases, and see the only problem in determining the logical form of that system of beliefs. In contrast, we argue that the difficulty has lain precisely in specifying what it is that different peoples respectively believe. Since we find the portrayals of the purported examples in both 'home' and 'away' cases seriously problematic, the question dividing the contestants in the logical case is at least asked prematurely. In other words, we argue that the use of formal logic in these contexts (in particular the notion of 'contradiction') underestimates the difficulties in achieving accurate formalisation of real life examples:

The irony is that formal techniques have very little value in the assessment of an everyday argument. Before these techniques can be applied to an argument in its natural habitat, the argument first must be identified by restating it as a sequence of declarative sentences that are to include all its premises and its conclusion, and then it must be clarified by reformulating it so as to make explicit its logical form. But the translation into a form to which logical techniques are applicable is at least as problematic as any problem that would be resolved by the use of formal methods, if indeed any problem about such an argument can ever be resolved by these methods. ${ }^{9}$

In our view, many crucial difficulties arise in the transition between informal reports of what is believed and the recasting of those beliefs in more formal structures. We show that it is through this process of translation into formal terms that the actual sense of the

${ }^{9}$ Don S. Levi, In Defense of Informal Logic (Dordrecht: Kluwer, 2000); p. 8. See also Ludwig Wittgenstein, Remarks on the Foundations of Mathematics, 3rd ed. (Oxford: Basil Blackwell, 1978); Gilbert Ryle, “Formal and Informal Logic” in Dilemmas (Cambridge: Cambridge University Press, 1954), pp. 111-129; Peter Winch, “Understanding a Primitive Society”, American Philosophical Quarterly 1: 304324; Harvey Sacks, Lectures on Conversation (Oxford: Blackwell, 1992).

Greiffenhagen, C. and W. Sharrock (2006). Logical relativism: logic, grammar, and arithmetic in cultural comparison. Configurations 14 (3), 275-301. 
described practices is lost or distorted. It then becomes questionable whether these cases feature any kind of contradiction, let alone manifest even the incipient presence of an alternative logic.

Note that we are not saying that the discipline or practice of formal logic may not be culturally relative, in the sense that it may be found in our culture but absent from others. However, even if formal logic has been developed in one culture, it is not really the whole culture which has done so. Rather, formal logic has typically been developed as part of some highly specialized practices - in particular, mathematics, law, and metaphysics ${ }^{10}$. Consequently, to speak of formal logic as 'Western logic' is potentially misleading, since it suggests that it is the whole Western culture that has led to the development of formal logic (rather than some specialized practices) and that therefore the practice of formal logic is an expression of the whole culture (rather than an aspect of a culturally distinctive specialist practice).

We think that there are good reasons to suspect that the relationship between 'logic' and 'culture' has been exaggerated, since if it were the case that each culture had a different underlying logic (where that logic is an expression of how members of that culture 'think'), then this should be easily documented and extensively demonstrated since it would be pervasively apparent. Almost anything that was done in either of two cultures marked by such logical differences would manifest the divergence between them and

\footnotetext{
${ }^{10}$ William Kneale and Martha Kneale, The Development of Logic (Oxford: Oxford University Press, 1962); p. 2.
}

Greiffenhagen, C. and W. Sharrock (2006). Logical relativism: logic, grammar, and arithmetic in cultural comparison. Configurations 14 (3), 275-301. 
strikingly so. In fact, if different cultures thought according to different logics, then crosscultural discussion and debate would seem very difficult ${ }^{11}$.

However, there are only a few empirical candidate examples on offer (which may itself suggest something suspect about the relativist project). We will focus on two. The first concentrates on the question whether the Chinese think more 'dialectically' than Westerners. The second concerns supposed contradictions 'institutionalised' in Zande beliefs about witchcraft. This example is dealt with more extensively, since it has played a crucial part in discussions about cultural relativism and because there has been a rather complex controversy over what the case relevantly shows. Our attempt is to show that these examples at best point to the existence of different practices - in the sense that one culture may have developed the game of Bridge, while another developed the game of Go (and others may not have developed either of them) - but that there is no licence from such examples for claims about the whole culture, in particular about whether one culture thinks more logically or rationally than another (since there are real difficulties in specifying whether there is a way in which 'a culture thinks').

\section{The Chinese}

In our view the most fundamental problem with logical relativism lies in underestimating the difficulties in understanding the sense of a culture’s beliefs. Guided by philosophical or theoretical interests, the analyst too quickly tries to render what it is that a culture

\footnotetext{
${ }^{11}$ See Brian Huss, "Cultural Differences and the Law of Noncontradiction: Some Criteria for Further Research, Philosophical Psychology 17:3 (2004): 375-389; p. 375.
}

Greiffenhagen, C. and W. Sharrock (2006). Logical relativism: logic, grammar, and arithmetic in cultural comparison. Configurations 14 (3), 275-301. 
believes in the terms of formal logic. Our aim is to show that this rendering (or redescription) is often misleading.

\section{Formalising contradictions}

We start with a study that investigates the question of whether Chinese thought is different from Western thought, because the Chinese seemingly do not subscribe to the law of non-contradiction (LNC). This question is tackled through a study of Chinese philosophical sayings:

One of the most fundamental principles of Aristotle's logic is the Law of NonContradiction. [...] For Aristotle, it is impossible for contradictories to be truly said of the same object at the same time [...] According to this, if not-P is a contradictory or contrary of $\mathrm{P}$, then a proposition like ' $\mathrm{S}$ is both $\mathrm{P}$ and notP' can never be true. Aristotle treats the Law of Non-Contradiction as a basic undemonstrable principle. For him, empirically, everyone follows this law when he or she is reasoning, and therefore the law is absolutely universal.

Surprisingly, Eastern philosophical traditions seem to take exception to the obvious truism. [...] In Chinese tradition, especially the School of Names, there are many propositions which look like violations of the Law of NonContradiction. Typical examples occur in the following quotations [...] (1) 'Each thing is the same as other things and yet each thing is different from other things.' (2) 'The heavens are as low as the earth, mountains are on the same level as marshes.' (3) 'The creature coming into life is the creature ceasing to be alive.' All these propositions can be formulated as ' $\mathrm{S}$ is both $\mathrm{P}$ and not-P, ${ }^{12}$

\footnotetext{
${ }^{12}$ Xinyan Jiang, "The Law of Non-Contradiction and Chinese Philosophy", History and Philosophy of Logic 13 (1992): 1-14; pp.1-2; our emphasis.
}

Greiffenhagen, C. and W. Sharrock (2006). Logical relativism: logic, grammar, and arithmetic in cultural comparison. Configurations 14 (3), 275-301. 
Jiang wants to show that although these propositions can be formulated as violations to Aristotle's LNC, alternative formulations are possible. Jiang moves from a binary logic (with truth values 0 and 1 ) to a continuous logic (with values ranging between 0 and 1). Rather than seeing 'black' and 'white' as polar opposites, we are then able to allow different degrees of 'blackness' and 'whiteness':

A famous example is Chuang Tzu's proposition 'A white dog is black' [...]. For Chuang Tzu, what is called a white dog is one whose colour is white much more than it is black. ${ }^{13}$

Using this alternative logic, Jiang is able to formalise propositions (1) to (3) in such a way that they do not express contradictions ${ }^{14}$. Jiang here follows a familiar line of argument in the studies that we have reviewed: according to one logic, the rendering of a sentence contains a contradiction, but according to another logic it does not. Does this not show that logic is relative?

At this point, we only want to highlight that Jiang treats remarks which plainly have very different senses (i.e., say very different things) as though they were instances of the same proposition (rather than sharing merely a superficially similar verbal form). “That dog is black and white all over" if said with the meaning "That dog is simultaneously black all over and white all over so that every point on its coat is both black and white" would be internally contradictory. "That dog is black and white all over", if said to express that the dog's coat had different colour patches but only those of black and white would not be.

\footnotetext{
${ }^{13}$ Ibid., p.7; our emphasis.

${ }^{14}$ For example, proposition $(1)$ is formalised as “ $(\exists \mathrm{n})[\mathrm{R}(\underline{\mathrm{F}})(\underline{\mathrm{a}})=\mathrm{n}, \mathrm{R}(\underline{\mathrm{non}}-\underline{\mathrm{F}})(\underline{\mathrm{a}})=1-\mathrm{n}$, where $0<\mathrm{n}<1$ and $\mathrm{n}$ $\neq 0.5] . ”($ Ibid., p.11)
}

Greiffenhagen, C. and W. Sharrock (2006). Logical relativism: logic, grammar, and arithmetic in cultural comparison. Configurations 14 (3), 275-301. 
What we are trying to highlight is the fact that the debate between universalists and relativists may arise out of the misreading of utterances with very different senses as expressing the same proposition. In fact, part of the problem originates in the tendency to treat every utterance as a proposition, whereas it is debatable whether proverbs, for example, are usefully thought of as propositions. As Sacks remarks in a lecture on 'proverbs':

It's a very usual use of proverbs among academics, to refer to them as 'propositions' and to suppose then that it goes without saying that the corpus of proverbs is subjectable to the same kind of treatment as, for example, is scientific knowledge. ${ }^{15}$

Consider the following famous proverbs by Sepp Herberger, a former coach of the German football team:

(1) „Der Ball ist rund.“ [“the ball is round”]

(2) „Nach dem Spiel ist vor dem Spiel.“ [“after the match is before the match”]

Similar witticisms are attributed to the American baseball player Yogi Berra (sometimes referred to as Yogisms):

(3) "It ain’t over till it’s over."

(4) "If the world were perfect, it wouldn't be."

(5) “It’s like déjà vu all over again.”

${ }^{15}$ Harvey Sacks, Lectures on Conversation (Oxford: Blackwell, 1992); p. 105.

Greiffenhagen, C. and W. Sharrock (2006). Logical relativism: logic, grammar, and arithmetic in cultural comparison. Configurations 14 (3), 275-301. 
It would be a simple mistranslation of these to treat (1) as a tautology ${ }^{16}$ and (2) as a contradiction - just as it would be to regard "Business is business" as a tautology rather than as, for example, a way of refusing to take any other considerations (such as those of friendship) into account in a business matter. Just because something can be translated into a formalism, does not mean that the formalism captures any intrinsic structure of that which is being paraphrased. Shanker ${ }^{17}$ reminds us of Wittgenstein's ${ }^{18}$ warning that the possibility of mapping Zulu war dances onto chess moves does not make those warriors tacit chess-players.

Harvey Sacks, in lectures on 'paradoxes'19, has remarked that people may deliberately exploit the fact that it is possible to formulate such paradoxical statements ${ }^{20}$, especially for the purposes of verbal humour ${ }^{21}$. In other words, in everyday life we encounter a number of 'paradoxical' statements, which are not treated as expressions of a logical mistake on the part of the speaker, but rather as expressing a profundity of sorts.

In sum, what are presented as violations of the Law of Non-Contradiction may seem to be such only as a result of the failure to faithfully translate the sense of 'informal'

\footnotetext{
${ }^{16}$ Sacks made some fine observations on 'tautological proverbs' (Ibid., Spring 1967, Lecture 13).

${ }^{17}$ Stuart G. Shanker, “AI at the Crossroads” in The Question of Artificial Intelligence: Philosophical and Sociological Perspectives, ed. Brian P. Bloomfield (London: Croom Helm, 1987), pp. 1-58; p. 39.

${ }^{18}$ Ludwig Wittgenstein, Philosophical Investigations (Oxford: Basil Blackwell, 1953); §200.

${ }^{19}$ Sacks, “Lectures” (above, n. 15), Spring 1966, Lecture 21; Fall 1967; Lecture 9.

${ }^{20}$ Ibid., p. 423.

${ }^{21}$ Ibid., p. 699.
}

Greiffenhagen, C. and W. Sharrock (2006). Logical relativism: logic, grammar, and arithmetic in cultural comparison. Configurations 14 (3), 275-301. 
expressions into their logical form (since it is only in this logical form that contradictions become 'visible').

\section{Avoiding the appearance of contradictions}

Peng and Nisbett ${ }^{22}$ also try to show that logic has a different place in Western and Asian cultures. Through a series of experimental studies they aim to show different attitudes towards the Law of Non-Contradiction [LNC] among Chinese and American students. The studies were summarised in the following way:

Five empirical studies showed that dialectical thinking is a form of folk wisdom in Chinese culture: Chinese participants preferred dialectical proverbs containing seeming contradictions more than did American participants. Chinese participants also preferred dialectical resolutions to social conflicts and preferred dialectical arguments over classical Western logical arguments. $^{23}$

The actual thesis seems to be that the Chinese are less bothered by contradictions, i.e., that they are not so concerned to avoid the appearance of contradicting themselves as Americans are. The research conducted in support of this thesis also seems to be intended to show that the Chinese are less ready to recognise contradictions, at least in the sense that they are less prone to regard disputing positions as incompatible. They are therefore accredited with 'dialectical' reasoning which, in one prominent manifestation, seems to consist in a greater willingness to see truth on both sides (or, perhaps, to exhibit a tendency to regard two contesting positions as not entirely but only partially true, and

\footnotetext{
22 Kaiping Peng and Richard E. Nisbett, “Culture, Dialectic, and Reasoning about Contradiction”, American Psychologist 54:9 (1999): 741-754.

${ }^{23}$ Ibid., p.741; our emphasis.
}

Greiffenhagen, C. and W. Sharrock (2006). Logical relativism: logic, grammar, and arithmetic in cultural comparison. Configurations 14 (3), 275-301. 
therefore as such not directly in contradiction with each other). When it comes to the arguments and studies, these differences are a matter of degree only - the Chinese are alleged to be somewhat less attached to the LNC than Americans ${ }^{24}$.

The first study concerned the preferences for dialectical proverbs (and thus connects to Jiang's study):

Study 1 was designed to show that dialectical thinking is reflected in Chinese folk wisdom, and that dialectical proverbs are more common in Chinese everyday language than in American everyday language and are more preferred by Chinese than by Americans. Examples of proverbs containing contradictions include "beware of your friends not your enemies", which contradicts the very definition of friendship, and the proverb "too humble is half proud", which explicitly contradicts the very meaning of the word "humble". ${ }^{25}$

We do not question whether one language may contain more proverbs of a certain kind than another. We want to interrogate the sense in which the proverbs feature contradictions and are reminded of the title of a paper, "Why Tall Pygmies are Short"26, as a good example of how the unwary might be led to suppose that certain forms of expression embody a violation of LNC. Something cannot be both $\underline{A}$ and Not- $\underline{A}$ at once. Tall is not short and short is not tall, so Pygmies are being asserted to be both tall and not

\footnotetext{
${ }^{24}$ Richard E. Nisbett, Kaiping Peng, Incheol Choi, and Ara Norenzayan, "Culture and Systems of Thought: Holistic Versus Analytic Cognition”, Psychological Review 108:2 (2001): 291-310; p. 301.

${ }^{25}$ Peng and Nisbett, “Culture, Dialectic, and Reasoning about Contradiction” (above, n. 22); p.744; our emphasis.

${ }^{26}$ Louis Narens, “Why Tall Pygmies Are Short”, Social Science Working Paper 13 (School of Social Science, University of California, Irvine, 1972).
}

Greiffenhagen, C. and W. Sharrock (2006). Logical relativism: logic, grammar, and arithmetic in cultural comparison. Configurations 14 (3), 275-301. 
tall, which is a glaring contradiction. Of course, nothing of the sort is involved, for an individual can be tall for a Pygmy whilst being short in comparison to other peoples (like all other Pygmies). Tall and short are relative terms, and their application depends upon the standard of comparison involved, so that an individual can be one thing according to one standard whilst not being that according to another.

Peng and Nisbett identify a couple of Chinese proverbs as 'dialectical', i.e., seemingly self-contradictory. "Too humble is half proud" says one, the other "beware of your friends but not your enemies" - but then it is easy enough to think of Western proverbs of a similar structure: "I can take care of my enemies but God must protect me from my friends" for one, and "one has to be cruel to be kind" for another. In any case, the proverbs do not involve a necessary contraction. Although they can be interpreted in such a way that they formulate a paradox, alternative interpretations are possible. For example, the first proverb does not say "humble is proud", but speaks of being too humble, which is excessive and therefore perhaps not genuine humility at all. An excessive, inauthentic humility might border on a form of vanity. Equally, the second proverb does not say “your friends are your enemies”, but could be interpreted as saying that friends can be dangerous to you in other ways than enemies can (which does not mean that a contradiction is involved in respect of the meaning of the words 'friend' and 'enemy’).

Finally, although there are proverbs that trade on puzzles, paradoxes, or contradictions (see the examples by Herberger and Yogi above), this does not mean that a culture which 'accepts' these proverbs therefore does not 'accept' the LNC. The seeming contradiction disappears as soon as one reflects upon what the proverbs want to say. Proverbs are not

Greiffenhagen, C. and W. Sharrock (2006). Logical relativism: logic, grammar, and arithmetic in cultural comparison. Configurations 14 (3), 275-301. 
straightforward propositions, but rather are better thought of as warnings, guidelines, anecdotes, etc., which are often formulated in a thought-provoking manner ${ }^{27}$.

The third study:

In Study 3, participants from both cultures were asked to write down what they thought about two everyday-life contradictions, including the origin and resolution of the two conflicts. Their responses were then coded as dialectical or nondialectical. [...] A dialectical response was defined as one that (a) addressed the issues from both sides and (b) attempted to reconcile the conflicts by comprising. [...] Nondialectical responses generally found exclusive fault with one side or the other $[\ldots]^{28}$

An example of an everyday-life contradiction was the following:

Mother-Daughter Conflict: Mary, Phoebe, and Julie all have daughters. Each mother has held a set of values which has guided her efforts to raise her daughter. Now the daughters have grown up, and each of them is rejecting many of her mother's values. How did it happen and what should they do? ${ }^{29}$

Again, we want to highlight that these are not contradictions in any logical sense, but rather, disagreements. They possibly point to different cultural values (e.g., the supposed greater importance of the family and of respect for elders in Chinese culture), but they carry no suggestion that anyone is embracing contradictions as such (and is therefore thinking 'dialectically'). Conflicts are not the same as contradictions (a point we will take up in the conclusion).

\footnotetext{
${ }^{27}$ See again Sacks, “Lectures” (above, n. 15) on 'proverbs’ (Fall 1964/Spring 1965, Lecture 13) and on the use of 'tautological proverbs' (Spring 1967, Lecture 13).

${ }^{28}$ Peng and Nisbett, “Culture, Dialectic, and Reasoning about Contradiction” (above, n. 22); p.746.

${ }^{29}$ Ibid., p. 753.
}

Greiffenhagen, C. and W. Sharrock (2006). Logical relativism: logic, grammar, and arithmetic in cultural comparison. Configurations 14 (3), 275-301. 
The fourth study contrasted two different kinds of argument for the existence of God:

The logical argument for the existence of God [...] uses the law of noncontradiction to argue 'the first causality' of the universe, suggesting that because everything has a cause, and a mere succession of causes and effects is impossible, there must be a first or ultimate cause of the universe, which must be God. The dialectical argument applying the principle of holism argues that when two people see a cup from opposite perspectives, one sees a cup with a handle, and the other sees a cup without a handle. But there must be a God above individual perspectives who can see all and who decides the truth. ${ }^{30}$

Again, we do not want to question whether Americans or Chinese may display a preference for one form of argument over another, but we wonder what this says about $\underline{\text { logic. As Huss }}{ }^{31}$ points out, many formulations of the First Cause Argument involve at least one premise that comes close to contradicting the conclusion, since one of the premises usually involves some claim to the effect that nothing can come to be without a cause, but the conclusion of the argument is that there must exist something without a cause. This means that the First Cause Argument, rather than testifying to an American preference for the LNC, would seem to point in the other direction, i.e., to exhibit an American preference for 'dialectical' arguments. The Americans could be seen to embrace (rather than to avoid) arguments which at least appear contradictory.

In sum, nothing in Peng and Nisbett's studies needs to be regarded as evidence for the proposition that the Chinese are demonstrably less attached to the LNC than Americans (only, possibly, as evidence that Chinese are more conciliatory than Americans, i.e., more

\footnotetext{
${ }^{30}$ Ibid., p. 747.

${ }^{31}$ Huss, “Cultural Differences and the Law of Noncontradiction” (above, n. 11); p. 384.
}

Greiffenhagen, C. and W. Sharrock (2006). Logical relativism: logic, grammar, and arithmetic in cultural comparison. Configurations 14 (3), 275-301. 
ready to accommodate both parties in a disagreement than to insist that one should prevail). The studies cannot evidence varying degrees of commitment to avoiding the appearance of contradiction, since the materials provided to provoke the research subjects’ reactions do not feature genuine contradictions.

Even more basically, questions can be put against the way in which these instances are meant to exhibit the more generalized proclivities of a culture, i.e., in which way can a few, very selective, examples be employed as evidence of how a whole culture 'thinks'? Were it the case that the proverbs to which they appeal should be construed in the way Peng and Nisbett do, this would still leave the question as to whether proverbs of this kind are only to be found in Chinese culture and in which way there are significant 'logical' differences from the structure of North American proverbs. And, of course, there is the big question: What would the form of proverbs show about how, for example, Americans generally think?

We have no in principle objection to psychological or anthropological comparisons, but question attempts to cast such comparisons in a form which wonders whether Westerners are in general much more inclined to acknowledge the law of the excluded middle than Chinese are. As already pointed out above, if this were the case, then substantiation should presumably be easy, since were such general contrasts in play, then anything from either culture should instantiate the differences. However, in reality it is actually difficult to imagine what would or could produce clear and decisive substantiation of those contrasts in anything like a precise form. In sum, while these studies may - possibly point to some cultural differences, they do not point to any systematic difference in 'logic'.

Greiffenhagen, C. and W. Sharrock (2006). Logical relativism: logic, grammar, and arithmetic in cultural comparison. Configurations 14 (3), 275-301. 


\section{The Azande}

The immensely well-rehearsed case of oracles, witchcraft, and magic as recorded by the anthropologist Evans-Pritchard ${ }^{32}$ is the prime example of the supposed logical heterogeneity of reasoning ${ }^{33}$. The debate concerns the question whether the Azande have a different logic than we do and does not seem to originate with Evans-Pritchard himself, but with other people's rendition of his anthropological study, for example, Bloor's section on 'Azande logic and Western science'34. Tellingly, the debate typically only focuses on a few pages (pp. 23-26) of Evans-Pritchard's enormous book ${ }^{35}$.

The argument centres on the question as to whether or not the Azande's witchcraft practices feature a contradiction. If it did, then this would seemingly show that the Azande have a wholly different rationality from us, since it is not merely that they do not

\footnotetext{
${ }^{32}$ E. E. Evans-Pritchard, "Witchcraft” (above, n. 2).

33 It was Peter Winch’s paper “Understanding a primitive society”, American Philosophical Quarterly 1 (1964): 304-324, which did so much to draw the attention of philosophy of social science to EvansPritchard's report. The original discussion - see, for example, Brian R. Wilson, ed., Rationality (Oxford: Blackwell, 1970); and Melvin Pollner, Mundane Reason: Reality in Everyday and Sociological Discourse (Cambridge: Cambridge University Press, 1987) - surrounded the "failures and inequalities of the poison oracle” (Evans-Pritchard, "Witchcraft” (above, n. 2), p.338). In contrast, logical relativists focus on Zande beliefs about witchcraft.

${ }^{34}$ Bloor, “Knowledge and Social Imagery” (above, n. 8), pp. 123-130.

${ }^{35}$ We concur with crucial points in the critique by Timm Triplett, in his “Azande Logic Versus Western Logic?”, British Journal for the Philosophy of Science 39:3 (1988): 361-366, and “Is There Anthropological Evidence That Logic Is Culturally Relative?: Remarks on Bloor, Jennings, and EvansPritchard”, British Journal for the Philosophy of Science 45:2 (1994): 749-760. Bloor's position was defended by Angus Gellatly, "Logical Necessity and the Strong Programme for the Sociology of Knowledge”, Studies in History and Philosophy of Science 11:4 (1980): 325-339, and Richard C. Jennings, “Zande Logic and Western Logic”, British Journal for the Philosophy of Science 40:2 (1989): 275-285.
}

Greiffenhagen, C. and W. Sharrock (2006). Logical relativism: logic, grammar, and arithmetic in cultural comparison. Configurations 14 (3), 275-301. 
recognise this contradiction, but that they do not grasp the idea of 'a contradiction'. Actually, it is not their witchcraft practice as a whole which is the focus of relativist debates about logic, but only one point within that practice: whether logic shows that in their witchcraft system every male member of a clan, in which witchcraft is identified for one member, should therefore be automatically identified as a witch as a logical consequence of the way in which the powers of witchcraft are inherited through the male line. Thus, in his original report, Evans-Pritchard states that "To our minds it appears evident that if a man is proven a witch the whole of his clan are ipso facto witches, since the Zande clan is a group of persons related biologically to one another through the male line.”36 He goes on to say that: “Azande see the sense of this argument but they do not accept its conclusions, and it would involve the whole notion of witchcraft in contradiction were they to do so. In practice they regard only close paternal kinsmen of a known witch as witches. It is only in theory that they extend the imputation to all a witch’s clansmen. ${ }^{\text {’37 }}$ Out of such slight materials is the potential presence of a distinctive logic (or even different rationality) conjured. In our view, the safest conclusion would be that, given the evidence, it is uncertain quite what the Zande do believe.

Evans-Pritchard's own central concern was with why the Azande did not grasp that their system did not really work (since it did not accord with the known principles of science). Evans-Pritchard, puzzled by the reluctance of the Azande to 'see' the contradiction, gives an explanation as to how the Azande manage to 'avoid' drawing his conclusion (that

\footnotetext{
${ }^{36}$ Evans-Pritchard, “Witchcraft” (above, n. 2), p. 24.

${ }^{37}$ Ibid., p. 24.
}

Greiffenhagen, C. and W. Sharrock (2006). Logical relativism: logic, grammar, and arithmetic in cultural comparison. Configurations 14 (3), 275-301. 
either all or no members of a clan are witches): "Further elaborations of belief free Azande from having to admit what appear to us to be the logical consequences of belief in biological transmission of witchcraft."38

Firstly, if a man is proven to be a witch, his kin may accept that claim, but deny that he is in fact a member of their clan (i.e., he is a 'bastard'). Secondly, the witchcraft-substance that is the source of the witches power, may be dormant ('cool') - and one only becomes a witch once one uses witchcraft: “Also Zande doctrine includes the notion that even if a man is the son of a witch and has witchcraft-substance in his body he may not use it. It may remain inoperative, 'cool’ as the Azande say, throughout his lifetime, and a man can hardly be classed as a witch if his witchcraft never functions.”39 Finally, the Azande are said not to perceive the contradiction, because they only have a practical, but not a theoretical, interest in the subject:

Azande do not perceive the contradiction as we perceive it because they have no theoretical interest in the subject, and those situations in which they express their beliefs in witchcraft do not force the problem upon them. A man never asks the oracles, which alone are capable of disclosing the location of witchcraft-substance in the living, whether a certain main is a witch. He asks whether at the moment this man is bewitching him. ${ }^{40}$

\footnotetext{
${ }^{38}$ Ibid., p. 24.

${ }^{39}$ Ibid., p. 25.

${ }^{40}$ Ibid., p. 25.
}

Greiffenhagen, C. and W. Sharrock (2006). Logical relativism: logic, grammar, and arithmetic in cultural comparison. Configurations 14 (3), 275-301. 
As pointed out in the introduction, there are at least two interpretations of this example. Some people ${ }^{41}$ have argued that there is a contradiction only if we evaluate Zande beliefs according to classical logic. If we use an alternative (e.g., many-valued) logic then there is no contradiction. The Azande thus reason as logically as we do - but according to a different logical system. Others ${ }^{42}$ pursue a symmetrical approach, pointing out that contradictions appear not only in Zande beliefs, but also in our (Western) beliefs. However, each culture typically fails to recognise such contradictions (and only recognises them in someone else's culture), since recognising contradictions in their 'home' culture would threaten their social institutions:

[...] if the Azande were to see the error then one of their major social institutions would be untenable. It would be under the thereat of being found contradictory or logically defective, and hence its survival would be endangered. In other words it is vital that the Azande maintain their logical error on pain of social upheaval and the need for a radical change in their ways. $^{43}$

Thus for Bloor there is a contradiction in Zande belief (in the attenuated sense that there is both a contradiction 'for us', but no contradiction 'for them'), but the Azande avoid seeing this contradiction by drawing "a few cunning distinctions”, ${ }^{44}$, namely the concepts

${ }^{41}$ For example, Cooper, “Alternative Logic in 'Primitive Thought”” (above, n. 6) and Raven, “The Enculturation of Logical Practice” (above, n. 4).

${ }^{42}$ For example, Bloor, “Knowledge and Social Imagery” (above, n. 8), Jennings, "Zande Logic and Western Logic” (above, n. 7), and Bruno Latour, Science in Action: How to Follow Scientists and Engineers through Society (Cambridge, MA: Harvard University Press, 1987).

${ }^{43}$ Bloor, “Knowledge and Social Imagery” (above, n. 8), p. 124.

${ }^{44}$ Ibid., p. 126.

Greiffenhagen, C. and W. Sharrock (2006). Logical relativism: logic, grammar, and arithmetic in cultural comparison. Configurations 14 (3), 275-301. 
of 'cool' witchcraft-substance and 'bastard' clan members. This, for Bloor, is evidence that the Azande, rather than being driven 'by logic', are mainly influenced by their social institutions: “[...] the really weighty factors are the two, socially taken-for-granted elements in the situation: the use of the oracle and the general innocence of the clan as a whole.”45 Bloor concludes: "Logic poses no threat to the institution of witchcraft, for one piece of logic can always be met by another."

This, however, does not mean that the Azande are inferior to us, since we also have ‘institutionalised’ logical mistakes. Both Bloor and Latour use the example of bomber pilots who are not classified as murderers, despite the fact that murderers are those who deliberately kill people - for a Zande anthropologist this might be a clear contradiction that 'we' (Westerners) fail to see. If the anthropologist were to point this out to us, we might make similar 'excuses' as the Azande. Consequently: “This all suggests that the Azande think very much as we do. Their reluctance to draw the 'logical' conclusion from their beliefs is very similar to our reluctance to abandon our common sense beliefs and our fruitful scientific theories."

The symmetrical logical relativist thus argues that the question whether a particular belief system is contradictory is relative, since one culture may decide that it is and another that it is not (Evans-Pritchard sees a contradiction while the Azande seemingly do not) and

\footnotetext{
${ }^{45}$ Ibid., p. 126.

${ }^{46}$ Ibid., p. 126.

${ }^{47}$ Ibid., p. 129.
}

Greiffenhagen, C. and W. Sharrock (2006). Logical relativism: logic, grammar, and arithmetic in cultural comparison. Configurations 14 (3), 275-301. 
there is no need to adjudicate between the two views, since they are not rivals with respect to a common situation, but independent parts of different cultural realities.

Both the alternative and the symmetric relativist assume there is a contradiction looming in the Zande belief system. But is there? We wonder whether it is only after the beliefs have been 'translated' into a series of propositions that the question arises whether there is, or there isn’t, a contradiction.

\section{The example reconsidered}

Logical relativists see a contradiction in Zande beliefs about witchcraft. This is presumably due to the second of the above quotations from Evans-Pritchard: “Azande see the sense of this argument but they do not accept its conclusions, and it would involve the whole notion of witchcraft in contradiction were they to do so.”48

What does Evans-Pritchard actually say in this quotation? He says that the Azande "see the sense" of the argument (when, presumably, he presented it to some of them). However, seeing the sense of an argument does not mean that one accepts the argument. For example, a friend of ours very convincingly argues that flying is currently a great danger to the environment. Consequently he does not fly anymore. We can see the sense of his argument, but both continue flying nevertheless.

Furthermore, even if there was a contradiction, it surely cannot be that because the Azande do on this one occasion contradict themselves that they must be generally different from us, since we all know that in a certain sense we sometimes contradict

${ }^{48}$ Evans-Pritchard, “Witchcraft” (above, n. 2), p. 24.

Greiffenhagen, C. and W. Sharrock (2006). Logical relativism: logic, grammar, and arithmetic in cultural comparison. Configurations 14 (3), 275-301. 
ourselves. Neither does the example demonstrate that the Azande contradict themselves all the time, for the reason why attention focuses only on this aspect of their witchcraft practices is (presumably) because it is exceptional in their life. In other words, the Azande do not go around saying things like "I’ll see you later, but I won’t see you later." or “I have a child, but I don’t have a child.” (though even if they did say such things they wouldn't necessarily be contradicting themselves, any more than someone who, when asked whether they like something, replies “Well, I do and I don’t.”).

It could possibly be argued that they acknowledge a contradiction in this specific case, but that they are not much bothered by it, since it makes no difference to their lives. This is perhaps what Evans-Pritchard means when he talks about the difference between 'in theory' and 'in practice', though, again, there is not enough detail to understand quite how the contrast works and applies (i.e., what it means to say that 'in theory' all male members of a clan are witches). However, even if there was a contradiction in this peculiar case, what would this show?

- Sometimes people contradict themselves.

- People do not always agree whether something is a contradiction.

- It is often hard to make the allegation of a contradiction stick, i.e., to get people to accept that they are contradicting themselves.

- That something involves a contradiction may not always matter (much, if at all) to the people affected.

All of these remarks apply both to the Azande and to 'us'. The only way that any implications could be drawn from this example would be if it could be shown while we

Greiffenhagen, C. and W. Sharrock (2006). Logical relativism: logic, grammar, and arithmetic in cultural comparison. Configurations 14 (3), 275-301. 
'see' a contradiction in Zande beliefs regarding witchcraft, the Azande are unable to see this contradiction (since their institutions 'blind' them from seeing the contradiction).

Note that when Evans-Pritchard remarks that "Azande see the sense of this argument but they do not accept its conclusions”, it is unclear whether the Azande accept the premises of Evans-Pritchard's argument whilst rejecting the conclusion - or whether they reject both premises and conclusion together. Again, the bugbear of converting what people ordinarily say into adequate logical formulations is manifest. Consider, for example, an instance from within our society: a wife might say "all men are unfaithful”, but might still deny that they her husband will be unfaithful ("he might be, if you gave him the chance, but I don't give him the chance”). In other words, it is not clear whether Evans-Pritchard is identifying the grounds the Azande themselves gave for rejecting the argument that appears evident to him (because it would be contradictory to the rest of their notion of witchcraft), or whether he is making an observation on his own part of the unfortunate consequences that would follow were they to do so.

Furthermore, note that Evans-Pritchard does not suggest that the Azande have voiced or embraced an explicit contradiction ${ }^{49}$. That is to say, the Azande do not claim:

- “All Azande men are witches.”; and

- “Not all Azande men are witches.”

The apparent contradiction is derived by Evans-Pritchard as 'implicit' in Zande expressions of what they believe. In other words, the Azande did not themselves utter or

\footnotetext{
49 Neither do we, pace Walt Whitman in Song of Myself: "Do I contradict myself? Very well then I contradict myself, (I am large, I contain multitudes.)”
}

Greiffenhagen, C. and W. Sharrock (2006). Logical relativism: logic, grammar, and arithmetic in cultural comparison. Configurations 14 (3), 275-301. 
notice this possible contradiction while talking to Evans-Pritchard; instead it was EvansPritchard who summarised their beliefs in such a way that the Zande beliefs regarding witchcraft could be seen to lead to a contradiction.

Nothing in the evidence shows that the Azande are unable to recognise contradictions generally, i.e., it seems that they see a difference between things which feature contradictions and things which don't. The example demonstrates that they can recognise the implications of the anthropologist's argument. They are not completely baffled by the anthropologist's talk of contradiction, as though they have never heard of such a thing before or can't understand what the anthropologist is trying to say, etc. The Azande (apparently) concede that were they to believe what the anthropologist ascribes to them, they would indeed be in a contradiction, but they do not believe that, and that is why their beliefs are not contradictory.

The Azande thus seem to be able understand what a contradiction 'is', i.e., they are apparently able to recognise that there would be a contradiction if Evans-Pritchard summary formulation of their beliefs was allowed to stand as an account of what they believe (they "see the sense of his argument"). The Azande are neither unable to follow Evans-Pritchard's logical argument nor are they indifferent to it - rather, they reject that argument. They (apparently) deny that there is a contradiction and explain that EvansPritchard's formulation of their beliefs involves a misunderstanding of their practice, in which it isn't possible to tell who is genuine kin ('bastards') and it is not the case that all people with witchcraft substance are witches, because the witchcraft power is 'cool' (inoperative) in some cases (one might also note that if they were all operating as witches, there would be no point in using the oracle to decide which ones were).

Greiffenhagen, C. and W. Sharrock (2006). Logical relativism: logic, grammar, and arithmetic in cultural comparison. Configurations 14 (3), 275-301. 
We would therefore argue that the example, carefully considered, shows that the Azande understand what a contradiction is and what it would be to hold contradictory beliefs, but reject the claim that in the relevant case they do contradict themselves. Two interpretations are possible:

(1) the anthropologist has correctly identified a contradiction, but the Azande cannot recognize ('see') that this is a contradiction;

(2) the anthropologist has misidentified Zande belief, i.e., there simply is no contradiction.

Logical relativists prefer (1), since it allows an invocation of a distinction between 'them' and 'us': ‘we' see a contradiction, while ‘they' do not. For example, Bloor claims that for us, "there really is a contradiction in the Azande views whether the Azande see it or not”50. We wonder whether it has been established that there is a contradiction in Zande beliefs, or, put differently, on which 'level' there is a contradiction. It is clearly the case that the Azande beliefs are different from ours (they believe in witchcraft, we don’t), but has it been established that there is a contradiction in Zande beliefs (or only that it is possible to (mis-)translate the beliefs into a series of propositions that contradict each other)?

\section{Avoiding contradictions}

Logical relativists of the 'alternative logic' persuasion typically argue that there is a contradiction among the Azande, but only if they reason according to classical logic. The

${ }^{50}$ Bloor, “Knowledge and Social Imagery” (above, n. 8), p. 124.

Greiffenhagen, C. and W. Sharrock (2006). Logical relativism: logic, grammar, and arithmetic in cultural comparison. Configurations 14 (3), 275-301. 
'symmetrical treatment' logical relativist argues that the Azande do not 'see' a contradiction in order to protect their social institutions (just as we supposedly do not see the contradiction in our beliefs about murderers and bomber pilots). So Jennings ${ }^{51}$, in his defence of Bloor, makes the following points:

- "I argue that from the naturalistic point of view, Zande logic is different from Western logic and that there is no contradiction in Zande thought."

- “[Evans-Pritchard] does not tell us that the Azande revise their beliefs so that they are consistent. Rather his concern is how they manage to avoid seeing the contradiction. [...] Evans-Pritchard sees a contradiction but also recognizes that the Azande do not see a contradiction, that the Azande just accept the premises and do not accept the conclusion.”53

- “The Azande have various beliefs which, for us, have certain consequences. But, for the Azande, they do not have these consequences. ${ }^{\text {,54 }}$

- "Bloor argues that for the Azande there is no contradiction.,"55

As we have already pointed out, the Azande are not described as stating an outright contradiction (“all Azande men are witches and not all Azande men are witches”), but the apparent contradiction is inferred by Evans-Pritchard. We would argue that the supposed

\footnotetext{
51 Jennings, “Zande Logic and Western Logic” (above, n. 7).

${ }^{52}$ Ibid., p. 275.

${ }^{53}$ Ibid., p. 281; emphasis in original.

${ }^{54}$ Ibid., p. 282; emphasis in original.

${ }^{55}$ Ibid., p. 283; emphasis in original.
}

Greiffenhagen, C. and W. Sharrock (2006). Logical relativism: logic, grammar, and arithmetic in cultural comparison. Configurations 14 (3), 275-301. 
'avoidance' of the contradiction is also inferred by the analyst, and is an artefact of Evans-Pritchard's method of reconstructing the sequence of Zande thought.

Evans-Pritchard's own problem was that, so far as he was concerned, Zande beliefs were patently false, and his self-imposed task was to explain why this falsity was not equally apparent to the Azande themselves. Evans-Pritchard argued that the beliefs about oracles were protected from empirical falsification by having a two-part structure: a fundamental set of general principles and a subsidiary set of beliefs, which explained away failures in the application of the general principles. It seems that something like the same idea is at work in his account of the Azande's failure to accept that their beliefs about witchcraft suffer fatal logical flaws. Thus, he writes as if the Zande belief system consists of a set of fundamental beliefs and a set of secondary qualifications on them, the latter having the function of sealing off the contradiction carrying implications ${ }^{56}$.

There is an implicit temporal order to Evans-Pritchard's argument (and its interpretation by Bloor and Jennings): the Azande first form certain beliefs (which the anthropologist sees as involving a contradiction), then discern the damaging implications of that contradiction, and finally, in 'reaction', develop certain arguments that close off the possibility of a contradiction ('bastards', 'cool' witchcraft-substance, etc.). However, if statements about 'bastards' and 'cool' witchcraft-substance are not seen as subsidiary to,

\footnotetext{
${ }^{56}$ It is difficult to avoid the conclusion that both Evans-Pritchard and Bloor ascribe a proto-scientific theory to the Azande, something akin to Lakatos's 'hard core' and 'protective belt' - see Imre Lakatos, "Falsification and the Methodology of Scientific Research Programmes”, in Criticism and The Growth of Knowledge, ed. Imre Lakatos and Alan. Musgrave (Cambridge: Cambridge University Press, 1970), pp. 91-196.
}

Greiffenhagen, C. and W. Sharrock (2006). Logical relativism: logic, grammar, and arithmetic in cultural comparison. Configurations 14 (3), 275-301. 
but on a par, with the 'original' beliefs, there would be no problem. In that sense, witchcraft-substance would be a necessary but not sufficient condition for someone being a witch. In other words, the Azande would believe that:

- everyone who is a witch has witchcraft-substance (“Azande believe that witchcraft is a substance in the bodies of witches”57); but

- not everyone who has witchcraft-substance is a witch.

Thus when Evans-Pritchard defines 'witch' in the introduction to his book as “a person whose body contains, or is declared by oracles or diviners to contain, witchcraftsubstance and who is supposed to practise witchcraft" ${ }^{, 58}$, the first part could be seen as the necessary condition (witchcraft-substance), the second part as the sufficient condition (practising).

With respect to the supposed contradiction, Bloor argues that the Azande avoid (or do not detect) the contradiction. This, of course, presumes that there is a contradiction (though, of course, in Bloor's own terms this could only be a contradiction 'for Bloor' or 'for us', since the reality of a contradiction resides in its recognition). In our view, Bloor slips between what Schütz ${ }^{59}$ calls the perspective of actors and analysts. Adopting the perspective of actors, whether something is a contradiction or not would simply be a reflection on the language or practice of that culture. For example, in our society people may say that there is a contradiction between individual freedom (you should be free to

\footnotetext{
${ }^{57}$ Evans-Pritchard, "Witchcraft"”, (above, n. 2), p.21.

${ }^{58}$ Ibid., p.9; our emphasis.

${ }^{59}$ Alfred Schütz, Collected Papers I: The Problem of Social Reality (The Hague: Martinus Nijhoff, 1962).
}

Greiffenhagen, C. and W. Sharrock (2006). Logical relativism: logic, grammar, and arithmetic in cultural comparison. Configurations 14 (3), 275-301. 
do whatever you want) and the protection of members of society (restricting what you can do). In that case, it would be actors who identify a situation as involving contradictory wishes or principles. However, that is not quite what Bloor and Jennings are doing with Evans-Pritchard's account. It is the analyst that here determines (on behalf of both 'them' and 'us') whether something does, or does not, constitute denial of a contradiction $^{60}$. Bloor argues that 'we' see a contradiction, but that the Azande do not, because they have concealed it from themselves. Both claims seem to be contestable. Firstly, the Azande do 'see' the possibility of a contradiction, since they appreciate the sense of Evans-Pritchard's suggestion, but they do to accept the argument's conclusions, since they do not accept the whole argument. Thus Bloor and Jennings seem to contradict (pun intended) Evans-Pritchard's own account ${ }^{61}$. Secondly, in which sense do 'we' see a contradiction? It is clear that Evans-Pritchard sees a contradiction, and so does Bloor. However, all this shows is that logically-minded anthropologists can see a contradiction by setting out the beliefs in a particular logical format. This does not show that every 'Westerner' who ever got in contact with the Azande saw a contradiction (rather than a strange practice).

Bloor wants to argue that the Azande are as rational as we are, because we both have contradictions institutionalised in our practices. Thus Bloor points out that the Azande

\footnotetext{
${ }^{60}$ Steve Woolgar, "Interests and Explanations in the Social Study of Science”, Social Studies of Science 11:3 (1981): 365-394, makes a similar argument against 'interest explanations' of the 'strong programme' in the sociology of scientific knowledge.

${ }^{61}$ See also Triplett, “Is There Anthropological Evidence That Logic Is Culturally Relative?” (above, n. 35), p. 375.
}

Greiffenhagen, C. and W. Sharrock (2006). Logical relativism: logic, grammar, and arithmetic in cultural comparison. Configurations 14 (3), 275-301. 
deny the (to us apparent) contradiction in their beliefs about witchcraft - but then Westerners too deny the supposed contradiction in our beliefs about bomber pilots (Westerners do not actually believe that all killers are murderers). In contrast, we doubt that the Azande are denying that their beliefs involve a contradiction; rather, they are denying that Evans-Pritchard has managed to render their beliefs.

\section{The place of logic}

We have been arguing that it is very common for anthropologists or sociologists to overlook the importance of the work that goes into describing empirical examples. Thus we have tried to show that in the case of Evans-Pritchard's description of Zande beliefs about witchcraft a lot depends on the way that the beliefs are formulated. In other words, the way in which logic is deployed in producing and formulating the description is indispensable to the (perhaps unfounded) possibility of a contradiction. The adequate evidential determination of whether a contradiction has been found depends upon both the input and the scruple with which that input handled. Logical considerations do not arise after the ways of a practice have been described, but are invoked in, and integral to, the description of the practice in the first place. The order of deliberations is not, and cannot be, one in which what the Azande believe is first set out, and then a decision made as to whether what they believe is contradictory or not. The considerations throughout pertain to establishing what it is the Azande believe (an exercise conducted, as we have pointed out, with a paucity of essential materials). Thus, we either can or cannot, from any given corpus of materials, decide whether the Azande definitively believe this (and do subscribe to a contradiction) or whether they believe that (and are therefore exempt from such a charge).

Greiffenhagen, C. and W. Sharrock (2006). Logical relativism: logic, grammar, and arithmetic in cultural comparison. Configurations 14 (3), 275-301. 
A purported logical formalisation of a practice can only be as good as the information on that practice. If the information does not accurately portray the practice, then a logical schematisation of that information cannot be a faithful portrayal of the logical order of that practice. It is also important not to conflate properties of the formalisation with properties of the formalised practice or belief. In other words, we have to be careful not to: "represent language by means of some system, such as the propositional calculus, and then mistakenly attribute to language itself what only properly belongs to that formal system.”62 Just because it is possible to identify contradictions in a purported schematisation of Zande witchcraft practices as a series of declarative sentences, does not mean that a genuine contradiction in the indigenous beliefs has been identified. One repeatedly comes back to the same question: Does this schematisation faithfully capture what they do believe?

The problem, as it is initially found, is one of deciding whether a given set of beliefs matches up with a standard logical template. However, the problem turns into one of deciding what the beliefs are in the first place and whether that problem is not with the beliefs themselves, but with the way in which the beliefs are prepared for logical assessment. For relativists, the objective does not seem to be, in any case, to exposit the form of these beliefs for their own sake, to recognise the limitations of the materials available for doing so, or to highlight to uncertainties and ambiguities in their initial anthropological characterisation. Instead, the beliefs are portrayed through the

\footnotetext{
${ }^{62}$ Richard McDonough, “The Mechanistic World View and the Myth of Logical Structure”, Iyyun: The Jerusalem Philosophical Quarterly 49 (2000): 23-59; p. 32.
}

Greiffenhagen, C. and W. Sharrock (2006). Logical relativism: logic, grammar, and arithmetic in cultural comparison. Configurations 14 (3), 275-301. 
discussants' assorted prior conceptions of the issues at stake, and the manner and consequentiality of attributing a contradiction.

\section{Conclusion}

Since we have been critical of logical relativism, we probably need to explain that we are not doing this on behalf of logical universalism. Surely enough, we are disputing whether there is a manifest contradiction in Zande religious practices and are suggesting that there may be no contradiction at all, but this is not because we want to maintain that the Azande and ourselves are alike in that we both think in terms of standard logic. We are actually arguing that neither of us is best characterised as thinking in terms of formal logic. For us, the kind of comparisons attempted by both universalists and relativists are suspect, since they overlay the portrayal of cultural practices with a set of a priori concerns and, in important respects, obscure the actual similarities and differences between 'them' and 'us'.

Ostensibly the overall comparison is of two different cultures by way of their individual comparison with the independent standard of logic, especially with the Law of NonContradiction and the Law of the Excluded Middle. However, we have been arguing that there is no genuine comparison, because the portrayal of the respective cultures is subordinate to the a priori concerns of the analysts with the status of logical laws. As a consequence, the cultural instances which are cited are entirely problematical as materials for the discussion that the analysts want to have. We have tried to show that in both of the cases considered, there are good reasons for declining the logical characterisations offered and for raising doubts as to whether there are any genuine contradictions in

Greiffenhagen, C. and W. Sharrock (2006). Logical relativism: logic, grammar, and arithmetic in cultural comparison. Configurations 14 (3), 275-301. 
beliefs involved. Even if such an exercise in logical comparison were a valid and worthwhile exercise, we would argue that much more would need to be done to prepare the ground for it in terms of the provision of secured descriptions of the cultural samples - though it is equally possible, as Don Levi intimates in the passage quoted above, that solution to the problem of providing sound descriptions of a culture's practice might also result in the evaporation of the supposed problem.

We have also argued that even if the chosen examples were soundly set out (i.e., thoroughly documented and unequivocal depictions of what is believed in respect of certain matters), there would still be considerable problems in seeing what those instances could signify with respect to the presence of generalised and uniform culturewide 'modes of thought' that are accurate and discriminating enough to be meaningfully contrasted. It is only in a context where assumptions are made about the wholesale form of a 'mode of thought' that the location of a single contradiction could possibly carry significant import vis-à-vis one culture or another.

We have identified two strategies for cultural comparison. The first of these assumes that standard logic is a good representation of how 'we' (in the West) think, but that there is a contest whether this representation identifies a set of universal standards that are the hallmark of rational thought. Insofar as other people's ways of thought deviates from ours, the question then arises whether this indicates a diminished level of rationality (a now outdated implication) or whether it exhibits the presence of an equal, but different rationality. If the latter, then standard logic ceases to be the sole arbiter of rational thought, and the possibility arises as to whether the rationality of other cultures is ensured by their compliance with a non-standard logic. However, both sides of this debate 
assume that some formal logical scheme is a good tool for capturing and portraying everyday practices of reason and argument. We have been drawing on arguments from the now well-established perspective of 'informal logic' which, to put it in its basic terms, doubts the value and effectiveness of mapping rather arbitrarily schematic formal devices onto instances of actual conduct - without first taking considerable steps to ensure that those instances are properly understood. Insofar as techniques of logical formalisation are intended to work on vernacular materials, it is an essential precondition of their application that the sense of the vernacular be properly specified. Premature or inappropriate moves toward logical formalisation can, as in the two cases considered here, only confuse and complicate matters.

The second strategy that we have identified argues that although there may be contradictions in other culture's beliefs, so there are contradictions in our beliefs. Our position might seem more in harmony with this case for symmetry, but this would also require the kind of wholesale comparisons that we argue are ill advised, since the question "Do they think in the same way that we think?” begs the question of whether there is a way in which 'we think' that can be abstracted from a host of different practices in which 'we' (heterogeneously) engage. With respect to the version of symmetry in hand, it seems to us that Bloor's argument simply avoids the questions about the proper understanding of what the Azande do believe. In its casual attitude to the examples both 'away' (witchcraft) and at 'home' (bomber pilots) it reinforces the point that the issues are not empirical ones to be decided through careful anthropological studies of cultural diversity, but are propelled by a priori conceptions about the standing of logic.

Greiffenhagen, C. and W. Sharrock (2006). Logical relativism: logic, grammar, and arithmetic in cultural comparison. Configurations 14 (3), 275-301. 
More fundamentally, there is reason for being dissatisfied with Bloor's treatment of the question of whether there is a contradiction. For reasons deriving from his 'strong programme’ proposals (which we obviously cannot debate here) Bloor is committed to the idea that in respect of 'knowledge' the appropriate sociological line is to assume that knowledge is what people regard as knowledge. However, this does not obviate prior and unresolved - questions, but only begs them, for Bloor's move involves a change in subject. Bloor's argument is that the difference between the Azande and their Western counterparts is that the former do not believe that they contradict themselves (which for Bloor shows that the Azande do not contradict themselves) whilst the latter believe that the Azande do contradict themselves (which for Bloor shows that the Azande do contradict themselves). This ostensibly establishes that the status of 'contradiction' is culturally relative - the Azande both do not, and do, contradict themselves, depending on which culture is being taken as the vantage point. However, the question "Do these people believe they contradict themselves?” is not the same question as, or substitutable for, “Is there a contradiction in the religious beliefs that these people hold?”. This second question is an indispensable prior to establishing what the substance of denials and affirmations of contradiction might be - what the contradiction being asserted and denied actually is - and this, as we have been stressing throughout, does not engage us in the logical adjudication of set of pre-determined beliefs, but in one of adequately specifying beliefs. Child ${ }^{63}$, in the very early days of the sociology of knowledge argued that its fundamental and neglected problem was that of 'imputation' of empirically adequate

${ }^{63}$ Arthur Child, “The Problem of Imputation in the Sociology of Knowledge”, Ethics 51:2 (1941): 200-219.

Greiffenhagen, C. and W. Sharrock (2006). Logical relativism: logic, grammar, and arithmetic in cultural comparison. Configurations 14 (3), 275-301. 
attributions of belief. In that respect, it may be that nothing very much has really changed in the intervening six decades.

In sum, cultural relativism is not the same as cultural diversity. Of course other cultures have many different practices from our culture (in fact, the existence of those practices allows us to identify them as 'other' cultures). Furthermore, these practices often seem strange to us since they seem to stand in conflict with the ways that we do things. However, being strange is not the same as being contradictory:

There is certainly conflict between European and Zande modes of thinking and even a sort of mutual exclusion; but this is not so far to say that they logically 'contradict' each other. It could be that people who interest themselves in cricket find it impossible to take baseball seriously, and vice versa: there would be conflict here too, but no contradiction. It would make little sense to ask, in the abstract, which game it was 'right' to support (though of course in particular circumstances a man might have reasons for supporting the one rather than the other) ${ }^{64}$

\section{Acknowledgements}

We would like to thank Mike Lynch and one anonymous reviewer for comments on an earlier draft. Christian Greiffenhagen thanks the British Academy for a Postdoctoral Fellowship and a Small Research Grant which supported some of his work on this paper. Wes Sharrock thanks the Arts and Humanities Research Board (AHRB) for an Innovation

\footnotetext{
${ }^{64}$ Peter Winch, “Language, Belief and Relativism”, in Contemporary British Philosophy (Fourth Series), ed. H. D. Lewis (London: George Allen \& Unwin, 1976), pp.322-337; p. 330.
}

Greiffenhagen, C. and W. Sharrock (2006). Logical relativism: logic, grammar, and arithmetic in cultural comparison. Configurations 14 (3), 275-301. 
Page 41 of 41

Award (grant number B/IA/AN10985/APN17690) to support the work reported in this paper.

Greiffenhagen, C. and W. Sharrock (2006). Logical relativism: logic, grammar, and arithmetic in cultural comparison. Configurations 14 (3), 275-301. 